\title{
Diener dreier Herren? Kantonalbehörden und die Vollzugsvielfalt der arbeitsmarktlichen Bestimmungen im schweizerischen Asylrecht
}

\author{
Markus SPÖRNDLI, Thomas HOLZER und Gerald SCHNEIDER
}

\begin{abstract}
Zusammenfassung
Einer der viel beschworenen Vorteile des Föderalismus ist die Flexibilität, mit der sich zentralstaatliche Anliegen den regionalen Gegebenheiten anpassen lassen. Wir untersuchen diese Vorstellung am Beispiel des Asylrechts. Ausgangspunkt ist die Überlegung, dass die Vollzugsdelegation an die Kantone zu Prinzipal-Agent-Problemen führt und die Kantonalbehörden zu Dienern dreier Herren macht - der Bundesverwaltung, dern einheimischen Bevölkerung und regionalen Interessengruppen. Quantitativ zeigt sich, dass die Restriktivität der kantonalen arbeitsmarktlichen Ausführungsbestimmungen des schweizerischen Asylgesetzes nicht primär von der Nachfrage nach Arbeitskräften, sondern vom Einfluss ausländerfeindlicher Kräfte in den Kantonen bestimmt ist. Die Praxis der Erteilung von Arbeitsbewilligungen an Asylbewerber hingegen steht in keinem Zusammenhang zur Restriktivität der Regelungen und orientiert sich speziell in der Romandie an der arbeitsmarktlichen Lage. Auch wenn sich der Einfluss von Sonderinteressengruppen nicht nachweisen lässt, tanzen die kantonalen Entscheidungsträger so zumindest auf zwei Hochzeiten.
\end{abstract}

\section{Einleitung ${ }^{1}$}

Neben der direkten Demokratie gehört der Föderalismus zu jenen Merkmalen schweizerischer Staatlichkeit, die nicht nur in der Bevölkerung, sondern

\footnotetext{
${ }^{1}$ Dieser Artikel entstand im Anschluss an eine Seminararbeit, die Markus Spörndli am Institut für Politikwissenschaft der Universität Bern verfasst hat. An der Datenerhebung war auch Natalie Jan beteiligt. Thomas Holzer und Gerald Schneider danken dem Schweizerischen Nationalfonds (NFP 39) und der Stiftung "Bevölkerung - Migration - Umwelt" (BMU) für finanzielle Unterstützung.
} 
auch in der Wissenschaft auf grösste Zustimmung stossen. ${ }^{2}$ Nüssli (1985: 1213) unterscheidet sechs Argumente, die in diesem Zusammenhang immer wieder auftauchen: Erstens ergänze der Föderalismus das liberale Prinzip der Gewaltentrennung in vertikaler Hinsicht. Zweitens biete er Schutz für Minderheiten und verkürze drittens die Distanz zwischen Bürger und Staat. Ein weiterer Vorteil bestehe darin, dass der Föderalismus mehr Möglichkeiten zur politischen Partizipation biete. Schliesslich ermögliche er auch Experimente im lokalen und regionalen Rahmen und leiste einen entscheidenden Beitrag zur gesellschaftlichen Integration. Angesichts all dieser Pluspunkte ist es nicht erstaunlich, dass der Föderalismus als Organisationsform für die Schweiz nicht wegzudenken ist. So heisst es etwa bei Linder (1996: 181), der Föderalismus gehöre gleichsam zu den Grundwerten und Grundstrukturen der Schweiz. Nach Neidhart (1992: 29) ist der Föderalismus sogar eine jener politischen Errungenschaften, welche die Schweiz als Staatsnation zusammenhalten.

Auch in der internationalen Literatur findet man Zustimmung zu diesem wesentlichen Organisationsprinzip. Kilper und Lhotta (1996: 56-61) liefern einen umfangreichen "Tugendkatalog" der gängigsten Rechtfertigungen des Föderalismus, der denjenigen von Nüssli (1985) noch übertrifft. Für Deuerlein (1972: 233) erwächst der Föderalismus aus dem Wunsch, die Wechselbeziehungen zwischen Individuum und Gesellschaft auszubalancieren, wobei das Recht des Individuums gewahrt und der Anspruch der Gesellschaft nicht geschmälert werden soll. Und in einer kritischen Auseinandersetzung mit dem Föderalismus helvetischer Prägung attestiert Hueglin (1995: 207) immerhin: "The delegation of central powers back to lower levels of government ... appears to be a promising corrective to the inefficiencies and inflexibilities of the federal duplication of government and administration." Diese Aussage ist unter anderem in bezug auf die Vollzugsdelegation bundesstaatlicher Grundsatzentscheide an die Kantone zu werten. Dieser unter dem Begriff "Vollzugsföderalismus" geläufige Mechanismus gilt als zentrales Element des schweizerischen Politikprozesses (Abromeit 1993; Germann 1986). ${ }^{3}$ Er öffnet jedoch zumindest ein Potential

\footnotetext{
${ }^{2}$ Bis in die späten siebziger Jahre erstreckte sich die Literatur über den schweizerischen Föderalismus auf historische und juristische Studien (Wälti 1996: 116f.). Frey (1977) und Tanner (1982) legten erste ökonomische Arbeiten vor, die der Suche nach einer optimalen Aufgabenteilung zwischen Bund und Kantonen gewidmet sind. Erst nach Germanns (1975) Forderung, sich von der Beschränkung auf die Institutionen zu lösen und die Implementationsaspekte zum Untersuchungsgegenstand des Forschungsdesigns zu machen, begann sich die Politikwissenschaft eingehend mit dem Vollzugsföderalismus helvetischer Prägung zu befassen.

${ }^{3}$ Eine treffende Umschreibung des Begriffs liefert Bussmann (1986: 39): "als Aufgabenverteilung zwischen Bund und Kantonen, bei welcher der Bund einen inhaltlichen Einfluss auf die Kantone nimmt, diesen aber noch ein gewisser Spielraum bei der Aufgabenerfüllung bleibt." Wir schliessen uns dieser Definition an und verwenden den Begriff Vollzugsföderalismus in diesem Sinne.
} 
für Ungleichbehandlung. ${ }^{4}$ Dies ist besonders im Asylrecht gravierend, weil die betroffenen Asylsuchenden über keine Exit-Option verfügen. Sie werden zufällig einem Kanton zugeteilt und können einer eventuellen Diskriminierung nicht durch Abwanderung in andere Stände begegnen, die gegenüber Ausländern aufgeschlossener sind.

Die neueren politikwissenschaftlichen Resultate bestätigen die angesprochenen Probleme und widersprechen dem Optimismus, den vorab Ökonomen und Juristen verbreiten. ${ }^{5}$ Bemängelt werden v.a. die fehlende Gesamtverantwortung für den Aufgabenvollzug (Faganini 1991) und das "föderalistische Schwarz-Peter-Spiel" (Bussmann 1986). Probleme werden nicht zuletzt dort gesehen, wo das Ausmass einer Aufgabe und die Möglichkeiten der Regulierungsbehörde in einem Missverhältnis zu einander stehen (Howse 1995; Leonardy 1996; Höpflinger und Wyss 1993). So kann im Asylrecht zwar der kantonale Vollzug der arbeitsmarktpolitischen Ziele gerechtfertigt werden. Diese sind aber nicht von den übergeordneten bundesstaatlichen Zielen (vorläufige Nichtintegration von Asylsuchenden und Attraktivitätsminderung der Schweiz als Immigrations- und Asylland) zu trennen.

Besonders die Studien im Rahmen des NFP 6 belegen zudem, dass die Kantone es in bestimmten Situationen verstehen, ein Bundesgesetz nicht nur wie vorgesehen an die spezifische Situation anzupassen, sondern es geradezu für ihre Zwecke zu instrumentalisieren. ${ }^{6}$ Linder (1994: 44) meint dazu: "the principle that 'federal law breaks cantonal law' does not mean that 'federal policy breaks cantonal policy'. The implementation of federal law through the cantons, therefore, is mostly a political question". Wälti (1996: 122-123) bezeichnet den schweizerischen Föderalismus in diesem Zusammenhang als administrativen Föderalismus und greift ein Argument von Bussmann (1986) und Delley (1984) auf, welche der schweizerischen Form der Implementation eine Doppelfunktion zusprechen: Einerseits reagieren die Vollzugsorgane auf Anliegen der Öffentlichkeit für Regulation, gleichzeitig aber auch auf die Interessen von organisierten Gruppen.

In diesem Artikel wollen wir diese Hypothese anhand des schweizerischen Asylrechts überprüfen. Speziell gehen wir der Frage nach, wie die Kantone ihre Freiheiten bei den arbeitsmarktlichen Regelungen für Asylsu-

\footnotetext{
${ }^{4}$ Darauf macht z.B. Koritansky (1987: 179) aufmerksam: "administrative decentralization can be a cover for inequalities and perhaps inequities that may exist between different administrative units".

${ }^{5}$ Stellvertretend für andere seien hier Frey und Bohnet (1993: 78) angeführt: "federal competition serves as a safeguard against decisionmakers taking unfair advantage of their discretionary power".

${ }^{6}$ Eine Zusammenfassung der Projekte findet sich in Linder (1987). Ähnliche Feststellungen treffen auch Kiswling-Näf und Knoepfel (1992).
} 
chende ausschöpfen. ${ }^{\top}$ Anhand von drei konkurrierenden politökonomischen Modellen untersuchen wir, ob die Restriktivität dieser Regelungen durch die Situation auf den kantonalen Arbeitsmärkten, den Einfluss von Interessengruppen oder die Haltung der Bevölkerung gegenüber Asylsuchenden bestimmt ist. ${ }^{8}$ Unsere Analysen zeigen, dass die Lage auf dem Arbeitsmarkt zwar sehr wohl eine Rolle spielt, aber dass gerade die Kantone mit einer überdurchschnittlichen Arbeitslosenquote tendenziell liberalere Regelungen implementiert haben, was den Intentionen des Gesetzgebers fundamental widerspricht. Für dieses Ergebnis sind allerdings die Kantone der Romandie verantwortlich. Bei den Deutschschweizer Kantonen allein steht die Restriktivität in keinem Zusammenhang zur Arbeitslosenquote. Der Einfluss der Interessengruppen ist vernachlässigbar. Je negativer die Bevölkerung hingegen gegenüber Asylsuchenden eingestellt ist, desto schärfer sind die administrativen Regelungen auf dem Arbeitsmarkt. Die Bedeutung der ausländerfeindlichen Grundstimmung erfährt zudem im Kalkül der Kantonalbehörden insofern eine Differenzierung, als zwischen der Restriktivität der Regelungen und der Erteilung von Arbeitsbewilligungen kein Zusammenhang besteht. Die Resultate der statistischen Analyse belegen vielmehr, dass die kantonalen Arbeitslosenquoten die Erteilung der Arbeitsbewilligungen bestimmen. In diesem Sinne sind die kantonalen Behörden Diener mindestens zweier Herren, der eigenen Bevölkerung einerseits und des zentralstaatlichen Gesetzgebers andererseits.

Nach einer Diskussion der rationalistischen Literatur zum Föderalismus skizzieren wir die Interaktionen zwischen der Stimmbevölkerung sowie den Bundes- und Kantonalbehörden als Prinzipal-Agent-Problem. Danach beschreiben wir die Freiheiten, welche die Kantone bei den arbeitsmarktlichen Regelungen für Asylsuchende geniessen. Anschliessend umreissen wir das Research Design und präsentieren die Ergebnisse unserer multivariaten Analyse. Der Artikel schliesst mit einer kurzen Diskussion der politischen Implikationen, die sich aus den Ergebnissen ableiten lassen.

\footnotetext{
${ }^{7}$ Besonders seit der Gesetzesrevision vom 22. Juni 1990 und durch die Einführung der Verordnung über die Begrenzung der Zahl der Ausländer (BVO) vom 6. Oktober 1986 haben die Kantone in diesem Bereich einen beträchtlichen Handlungsspielraum.

${ }^{8}$ Wie in anderen Artikeln zu unserer Evaluation der europäischen und schweizerischen Asylpolitik legen wir unserer Analyse politökonomische Modelle zugrunde, in denen das Verhalten der verantwortlichen Behörden und Politiker eine formale Fundierung erfährt (Holzer, Schneider und Widmer 1997, 1998; Holzer und Schneider 1997).
} 


\section{Prinzipal-Agent-Probleme des Föderalismus und das Schweizer Asylrecht}

Eine der Ironien der wirtschaftswissenschaftlichen Literatur zum Föderalismus besteht darin, dass neuere Analysekonzepte dieser Disziplin nur zum Teil rezipiert sind. ${ }^{9}$ Das überrascht insofern, als die Machtteilung zwischen regionalen und zentralstaatlichen Akteuren an sich ein klassisches Prinzipal-AgentProblem darstellt. In normativer Hinsicht dienen die entsprechenden SenderEmpfänger-Modelle dazu, optimale Formen der Aufgabenteilung zwischen einem Auftraggeber (Prinzipal) und einem Auftragerfüller (Agent) zu ermitteln. ${ }^{10}$

Prinzipal-Agent-Modelle haben die politikwissenschaftliche Forschung in den letzten Jahren enorm beeinflusst. Beispiele hierfür sind etwa in der Kriegsursachenforschung (Bueno de Mesquita and Lalman 1992) und in der Integrationsforschung (Schneider 1997) zu finden. In dieser Perspektive besteht das erste grundlegende Merkmal des Vollzugsföderalismus darin, dass eine zentrale Behörde als massgeblicher juristischer Prinzipal Aufgaben an regionale Akteure delegiert. Der Lohn für solche Aufträge besteht dabei darin, dass den Kantonal- oder Länderbehörden im Austausch dafür ein gewisser Interpretationsspielraum zugestanden wird. In politökonomischer Perspektive bedeutet dies, dass der Agent diskretionäre Macht erhält. Das zweite Charakteristikum der Machtdelegation an subterritoriale Akteure ist, dass die kantonale Bevölkerung und regional organisierte Interessengruppen diese Freiheiten wiederum begrenzen und eine für sie optimale Vollzugslösung verlangen. Wenn zwischen den Interessengruppen und dem Stimmvolk Präferenzdivergenzen auftreten, sind Kantonalbehörden demnach Diener dreier Herren - der zentralstaatlichen Aufsichtsorgane, der eigenen Bevölkerung sowie regionaler Interessengruppen.

In dieser komplexen Abhängigkeit kann die kantonale Regierung nun grundsätzlich einem dieser drei Pole besondere Bedeutung schenken. Erstens ist es möglich, dass sie sich einzig nach den Interessen der Bundesbehörden ausrichtet und entsprechend dem Geist der zentralstaatlichen Bestimmungen nachlebt. Das hier zunächst skizzierte Sachwalter-Modell postuliert, dass sich die Regierung einzig an den objektiven Kriterien des Arbeitsmarktes ausrichtet. Konkret wäre die Verordnung über die Begrenzung der Zahl der Ausländer (BVO) die Richtschnur für das behördliche Verhal-

\footnotetext{
9 Ausnahmen nehmen sich der Budgetmaximierung subterritorialer Regierungen an. Eine aktuelle Diskussion der wichtigsten Literatur sowie ein formales Modell finden sich in Abizadeh und Cyrenne (1990) Ökonomische Modelle über den optimalen Zentralisierungsgrad einer Volkswirtschaft werden unseres Wissens nur losgelöst von der Prinzipal-Agent-Problematik diskutiert. Ein guter Überblick über ersteres findet sich in Frey und Kirchgässner (1994: 55-68), zur formalen Behandlung von letzterem siehe Kreps (1990: 577-719) oder Tirole (1988: 51-55).
} 
ten. Dies äusserte sich darin, dass sich die Restriktivität der arbeitsmarktlichen Bestimmungen an der kantonalen Arbeitslosigkeit auszurichten hätte. Eine erste Hypothese lautet entsprechend:

\section{Hypothese 1 ("Sachwalter-Modell")}

Je höher die Arbeitslosenquote in einem Kanton ist, um so restriktiver sind die Regelungen für die Erteilung von Arbeitsbewilligungen für Asylsuchende und desto niedriger fällt die Erwerbsquote für Asylsuchende aus.

Dabei kann sowohl die aktuelle als die vergangene Arbeitslosigkeit eine Rolle spielen. Die aktuelle Arbeitslosigkeit dürfte die informelle Praxis beeinflussen, mit der die Behörden den Arbeitsmarkt zu regulieren versuchen. Da sich Vollzugsbestimmungen aber nicht sofort automatisch nach der Konjunkturlage zum Untersuchungszeitpunkt (1995) richten können, könnte auch die wirtschaftliche Situation bei der Inkraftsetzung der Verordnung auf Bundesebene im Jahr 1990 eine Rolle gespielt haben. Wir werden beide Varianten der Sachwalter-Hypothese testen. Wir erwarten, dass sich zumindest die kantonalen Arbeitslosenquoten aus dem Jahre 1990 auf den Restriktivitätsgrad niederschlagen. Bei der Arbeitslosenquote von 1995 sollte der gleiche Einfluss nachzuweisen sein, wenn auch weniger deutlich. Darüber hinaus ist denkbar, dass die Arbeitslosenquote in bestimmten Wirtschaftszweigen, in denen Asylsuchende traditionell gute Erwerbsmöglichkeiten vorfinden, ins Kalkül der Behörden einfliesst. Wir werden bei der Überprüfung des Sachwalter-Modells auch diesem Umstand Rechnung tragen.

Wenn das Handeln der kantonalen Behörden aus politökonomischer Perspektive betrachtet wird, scheint die Orientierung allein an der Arbeitslosigkeit eher naiv. Der Public Choice-Ansatz und andere Spielarten der rationalistischen Politiktheorie unterstellen vielmehr, dass sich die Behörden an den Anliegen von speziellen Interessengruppen orientieren. Demnach spielt es eine Rolle, ob sich die Kantone bezüglich ihrer Wirtschaftsstruktur bzw. der damit verknüpften Interessenartikulation unterscheiden. Der Interessengruppen-Ansatz postuliert entsprechend, dass Sonderinteressen privilegierter Gruppen die Ausgestaltung der Vollzugsbestimmungen beeinflussen.

Ein solches Modell hat zunächst Money (1997) für die Migrationspolitik ausgewählter OECD-Staaten skizziert. Sie postuliert, dass sich unter den gesellschaftlichen Kräften sowohl Anhänger einer liberalen wie einer restriktiven Politik gegenüber Immigranten formieren. Je nach Durchsetzungskraft der einen oder anderen Seite setzen sich die staatlichen Stellen für eine Verhärtung des migrationspolitischen Kurses ein. Dabei sind nach Money auf der Nachfrageseite die lokalen Arbeitsmärkte und Wirtschaftsstrukturen entscheidend. Besonders Arbeitgeber im ersten und dritten Sek- 
tor verlangen nach einer Liberalisierung der Vollzugspolitik, wenn ein Nachfrageüberhang nach wenig qualifizierten, billigen Arbeitskräften besteht. Nach Money entsteht diese Forderung deshalb, weil in diesen Sektoren ein Kapitalexport oft schwierig ist. Dieser Trend wird auf der anderen Seite eventuell dadurch aufgewogen, dass dem Druck zugunsten einer liberalisierten Politik die Furcht entgegensteht, den die lokalen Arbeitskräfte vor einem Verlust ihres Arbeitsplatzes haben könnten. Entsprechend würde sich bei einer Vergrösserung der Arbeitslosigkeit in Kantonen, die einen überdurchschnittlichen Bedarf an Arbeitskräften mit geringer Qualifikation ausweisen, der Restriktivitätsgrad erhöhen. Umgekehrt gilt, dass in Ständen mit geringer genereller Arbeitslosigkeit dieser Trend in die umgekehrte Richtung zeigen sollte. Dies führt uns zu folgender Hypothese für das Interessengruppen-Modell.

\section{Hypothese 2 ("Interessengruppen-Modell")}

Je wichtiger in einem Kanton die arbeitsintensiven und unterdurchschnittlich produktiven Wirtschaftszweige sind, desto liberaler ist dessen Politik zur Erteilung von Arbeitsbewilligungen für Asylsuchende und desto höher liegt deren Erwerbsquote.

Nicht nur wirtschaftliche Interessengruppen können als intermediäre Organisationen Druck auf die kantonalen Behörden ausüben. Nach einem einfachen Downs 'schen Ansatz ist davon auszugehen, dass die mit der Asylpolitik befassten Regierungsräte zunächst einmal um ihre Wiederwahlchancen bangen. Deshalb sind sie hellhörig für das Ausmass der Fremdenfeindlichkeit, das in ihrem Kanton auftritt.

Dabei spielt nicht nur die politische Kultur, sondern auch das institutionelle Gefüge in den einzelnen Kantonen eine Rolle. In der Schweiz artikulieren vor allem die Schweizerische Volkspartei (SVP) und die Parteien der extremen Rechten dumpfe Vorurteile gegenüber Asylbewerbern. Eine Abschwächung erfährt der Zusammenhang dann, wenn ein Kanton gesellschaftlich multikulturell orientiert ist. Es ist davon auszugehen, dass sich Asylsuchende in Ständen mit einem hohen Ausländeranteil eher integrieren können und der Bevölkerung weniger auffallen. Ähnliche Zusammenhänge gelten zumindest im Vergleich von OECD-Staaten. Wie die Panelregressionen in Holzer und Schneider (1997) zeigen, weisen Staaten mit einem hohen Ausländeranteil eine grössere Nachfrage nach Asyl aus. Tendenziell sind diese Staaten auch durch eine grössere Aufnahmebereitschaft gegenüber Asylsuchenden gekennzeichnet.

Hypothese 3 ("Wiederwahl-Modell") 
Je ausgeprägter die Vorbehalte in einem Kanton gegenüber Asylsuchenden sind, desto restriktiver ist dessen Politik zur Erteilung von Arbeitsbewilligungen für Asylsuchende und um so niedriger fällt deren Erwerbsquote aus. Dieser Effekt kann durch einen hohen Ausländeranteil abgeschwächt werden.

Zusammenfassend erforschen wir die folgenden Analysemodelle:

Grafik 1: Sachwalter-, Interessengruppen- und Wiederwahl-Model

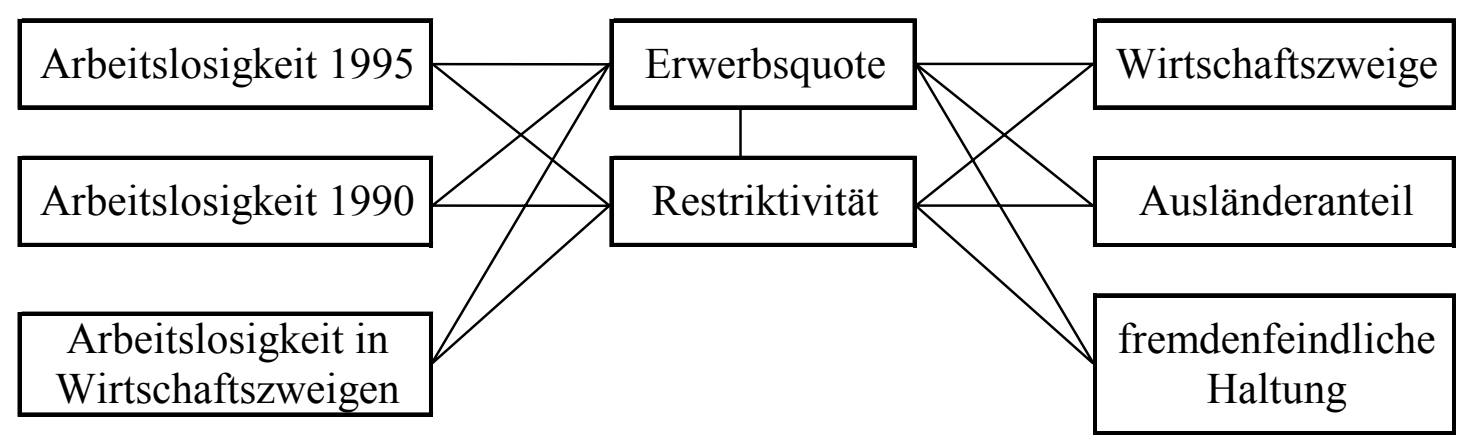

In der Mitte von Grafik 1 befinden sich unsere beiden abhängigen Variablen; links davon stehen die unabhängigen Variablen, die wir zur Überprüfung des Sachwaltermodells brauchen. Auf der rechten Seite sind die unabhängigen Variablen zur Überprüfung des Interessengruppen- bzw. Wiederwahlmodells dargestellt.

\section{Der Spielraum der Kantone}

Das am 5. Oktober 1979 verabschiedete und auf Anfang 1981 in Kraft getretene Asylgesetz (AsylG) galt als rechtlich verankerte Fortführung der bis dahin relativ liberalen Asylpolitik der Schweiz. In Artikel 21 wird denn auch der Grundsatz vertreten, dass jedem Asylbewerber die unselbständige Erwerbstätigkeit zu bewilligen sei. Angesichts der wachsenden Zahl an Asylgesuchen gerieten die Bundesbehörden aber bald unter Druck, so dass diese liberale Grundhaltung sukzessive aufgegeben wurde. Die verschiedenen Revisionen des Asylgesetzes umfassten dabei immer auch die Bestimmungen, mit denen die Möglichkeiten zur Arbeitsaufnahme für Asylsuchende geregelt sind. In der ersten Gesetzesrevision vom 16. Dezember 1983 wurde der Grundsatz in eine Ermessensvorschrift abgeschwächt. Die zweite Revision vom 20. Juni 1986 erlaubte den Kantonen die Einführung eines generellen dreimonatigen Arbeitsverbots für Asylsuchende. Am 22. Juni 1990 schliesslich wurde das Arbeitsverbot für verbindlich erklärt. Die 
Kantone haben allerdings die Kompetenz, das Arbeitsverbot um weitere drei Monate zu verlängern, falls innerhalb der ersten drei Monate ein negativer erstinstanzlicher Entscheid ergeht. ${ }^{11}$ Die restlichen in der Kompetenz der Kantone liegenden Instrumente sind in der Verordnung über die Begrenzung der Zahl der Ausländer (BVO) vom 6. Oktober 1986 enthalten. Nach Piguet und Misteli (1996: 79ff.) sind dort folgende vier Dimensionen besonders relevant. ${ }^{12}$ Erstens können die Kantone das in Art. 7 BVO verankerte Prinzip, wonach inländischen Arbeitnehmern ein Vorrang zukommt, in unterschiedlicher Intensität anwenden. ${ }^{13}$ Zweitens können sie generell, nur für einzelne Branchen oder gar nicht verlangen, dass der Arbeitgeber Bemühungen ausweist, "alle zumutbaren Anstrengungen unternommen zu haben, um eine Arbeitskraft auf dem inländischen Arbeitsmarkt zu finden". Drittens können die Kantone die Erteilung von Arbeitsbewilligungen an Asylsuchende auf einzelne Branchen beschränken, und viertens verfügen die Kantone diskretionäre Macht darüber, wie lang die Zeitperiode sein muss, bis ein Arbeitnehmer die Berufsbranche wechseln kann. Diese vielfältigen kantonalen Handlungsoptionen lassen erahnen, dass sich in der helvetischen Landschaft unterschiedliche Kombinationen der verschiedenen Instrumente etabliert haben. In der Tat wird von den Ständen der ganze Handlungsspielraum ausgeschöpft. Die Spannweite reicht vom Kanton Waadt, der für alle fünf Instrumente (die Verlängerung des Arbeitsverbots mitgerechnet) die liberalste Variante gewählt hat, bis zu den Kantonen Glarus und Appenzell Innerrhoden, die sich überall für die restriktivste Ausgestaltungsform entschieden haben. Basierend auf diesen fünf Instrumenten haben Piguet und Misteli (1996) und Spörndli (1997) einen Index für die Restriktivität erstellt. Im nächsten Kapitel diskutieren wir diese Operationalisierung.

\section{Definition der Variablen und Datenlage}

Zur Überprüfung unserer drei Hypothesen benötigen wir ein Mass für die Restriktivität bei der Erteilung von Arbeitsbewilligungen an Asylsuchende

\footnotetext{
${ }^{11}$ Vgl. Art. 21 Abs. 1 und 3 AsylG.

${ }^{12}$ Die von zwei Mitarbeitern des Forum suisse pour l'étude des migrations verfasste Arbeit stellte die Auftraggeberin, das Bundesamt für Flüchtlinge, zunächst einem nur sehr begrenzten Personenkreis zur Verfügung. Einer der Mitverfasser der vorliegenden Studie erhielt beispielsweise die Aufforderung, der solide Arbeitsbericht der zwei Neuenburger Forscher dürfe im Rahmen eines Universitätsseminars nicht an Studierende abgegeben werden.

${ }^{13}$ Wie aus dem Bericht über Konzeption und Prioritäten der schweizerischen Ausländerpolitik der neunziger Jahre hervorgeht, rangieren "Asylbewerber" und "vorläufig Aufgenommene" (soweit sie nicht Konventionsflüchtlinge sind) in der Hierarchie der Bundesbehörden an letzter Stelle. Der Grund für diese offiziöse Diskriminierung besteht darin, dass andernfalls das Ziel einer ausgeglichenen, stabilen Beschäftigungslage aus dem Auge rücken und eine andere Einstufung "die Attraktivität der Schweiz als Zielland irregulärer Wanderungen massiv anheben würde” (BIGA/BFA 1991: 49).
} 
in den einzelnen Kantonen. Piguet und Misteli (1996: 91f.) haben aus den fünf oben erwähnten Instrumenten einen Index der Restriktivität für die elf von ihnen untersuchten Kantone erstellt. Spörndli (1997) hat mit einem standardisierten Fragebogen, der an die zuständigen Stellen aller Kantone verschickt wurde, eine Neuerhebung durchgeführt. Wir stützen uns in unserer Analyse auf diese Erhebung, die den im März 1997 aktuellen Stand der Regelungen in allen Kantonen enthält. Wir operationalisieren den Restriktivitätsgrad in Analogie zu Piguet und Misteli (1996). Dieser Index berechnet sich als Summe der Restriktivitätsgrade der einzelnen Instrumente. Diese wurden nach dem folgenden Schema kodiert:

- ARBVERB: Arbeitsverbot nach Art. 21 AsylG (6 Monate = 3, 3 Monate = 0);

- BVO: Anwendung des Prinzips "Vorrang inländischer Arbeitnehmer" nach Art. 7 BVO (integral = 3, teilweise $=1.5$, keine $=0$ );

- BEMAG: Bemühungen der Arbeitgeber (verlangt =3, für gewisse Branchen verlangt $=1.5$, nicht verlangt $=0)$;

- BRAREG: Branchenregelung (vorhanden $=3$, nicht vorhanden $=0$ );

- BRAWE: Branchenwechsel (nicht möglich = 3, nach 24 Monaten möglich $=1.5$, nach 12 Monaten möglich $=1$, sofort möglich $=0$ ).

Beim Arbeitsverbot wurden auch Zwischenwerte verwendet. Tabelle 1 fasst die arbeitsmarktlichen Regelungen der Kantone zusammen. In der letzten Spalte wurde aufgrund des Restriktivitätsgrades eine Zuteilung der Kantone in drei Kategorien vorgenommen (RESKAT).

Als Indikator für die effektiv erteilten Arbeitsbewilligungen an Asylsuchende verwenden wir die kantonalen Erwerbsquoten von Asylsuchenden und vorläufig Aufgenommenen im August 1995. Wir bedienen uns der von Piguet und Misteli (1996: A31) zusammengetragenen Werte. Uns ist durchaus bewusst, dass die Gutheissungsquote der beantragten Bewilligungen theoretisch eine bessere Operationalisierung der Praxis bei der Erteilung von Arbeitsbewilligungen wäre. Erstens sind diese Angaben aber nicht verfügbar, und zweitens hat die Untersuchung von Spörndli (1997) ergeben, dass potentielle Arbeitgeber vor der Einreichung eines Gesuchs oft auf informellem Weg die Chancen einer Gutheissung abzuklären versuchen. Das führt dazu, dass nur Gesuche gestellt werden, denen eine gute Chance zur positiven Beurteilung eingeräumt wird. Die Gutheissungsquoten dürften deshalb stark nach oben verzerrt oder sogar einigermassen nivelliert sein und wären deshalb in der Praxis kein geeigneter Indikator zur Überprüfung unserer Hypothese. Piguet und Misteli (1996) haben sog. kontrollierte Erwerbsquoten berechnet, in denen sie die Einflüsse in der Zusammensetzung der Population der Asylsuchenden eines Kantons kontrollieren. Im Kanton Glarus bspw. sind junge Männer im Vergleich zu anderen Kantonen übervertreten. Dies führt dazu, dass die effektive Erwerbsquote in diesem Kanton eine der höchs- 
ten ist. Kontrolliert man für diesen Einfluss, so rutscht Glarus ins Mittelfeld ab. In unserem theoretischen Rahmen interessiert uns aber die tatsächlich erteilte Anzahl an Bewilligungen als Anteil an der Gesamtpopulation. Wir verzichten deshalb auf die Verwendung der kontrollierten Erwerbsquoten. ${ }^{14}$

Tabelle 1: Ausprägung und Zusammensetzung der Variable "Restriktivitätsgrad" (RESGRAD) in den schweizerischen Kantonen, geordnet nach Rangfolge; Trichotomisierung in RESKAT

\begin{tabular}{|c|c|c|c|c|c|c|c|}
\hline Kantone & ARBVERB & BVO & BEMAG & BRAREG & BRAWE & RESGRAD & RESKAT \\
\hline VD & .00 & .00 & .00 & .00 & .00 & .00 & .00 \\
\hline GE & .00 & .00 & .00 & .00 & 1.00 & 1.00 & .00 \\
\hline GR & 2.00 & .00 & .00 & .00 & 1.00 & 3.00 & .00 \\
\hline $\mathbf{L U}$ & .00 & 3.00 & .00 & .00 & .00 & 3.00 & .00 \\
\hline FR & .00 & 1.50 & 1.50 & .00 & 1.00 & 4.00 & .00 \\
\hline $\mathbf{N E}$ & .00 & 3.00 & .00 & .00 & 1.00 & 4.00 & .00 \\
\hline BS & .00 & 1.50 & 1.50 & 3.00 & .00 & 6.00 & 1.00 \\
\hline SZ & .00 & 1.50 & 1.50 & 3.00 & .00 & 6.00 & 1.00 \\
\hline TI & .00 & 3.00 & 3.00 & .00 & .00 & 6.00 & 1.00 \\
\hline $\mathbf{J U}$ & 1.50 & 3.00 & 3.00 & .00 & .00 & 7.50 & 1.00 \\
\hline OW & .00 & 1.50 & 3.00 & 3.00 & .00 & 7.50 & 1.00 \\
\hline SG & .00 & 1.50 & 3.00 & 3.00 & .00 & 7.50 & 1.00 \\
\hline $\mathbf{A R}$ & 2.00 & 3.00 & 3.00 & .00 & .00 & 8.00 & 1.00 \\
\hline NW & 2.00 & .00 & .00 & 3.00 & 3.00 & 8.00 & 1.00 \\
\hline TG & 3.00 & 3.00 & 3.00 & .00 & .00 & 9.00 & 1.00 \\
\hline BL & .00 & 3.00 & 3.00 & 3.00 & 1.00 & 10.00 & 1.00 \\
\hline VS & .00 & 3.00 & 3.00 & 3.00 & 1.00 & 10.00 & 1.00 \\
\hline SO & .00 & 3.00 & 3.00 & 3.00 & 1.50 & 10.50 & 1.00 \\
\hline BE & 2.00 & 3.00 & 3.00 & 3.00 & .50 & 11.50 & 2.00 \\
\hline AG & 3.00 & 3.00 & 3.00 & 3.00 & .00 & 12.00 & 2.00 \\
\hline UR & 3.00 & 3.00 & 3.00 & .00 & 3.00 & 12.00 & 2.00 \\
\hline ZG & 2.00 & 3.00 & 3.00 & 3.00 & 1.00 & 12.00 & 2.00 \\
\hline SH & 2.00 & 1.50 & 3.00 & 3.00 & 3.00 & 12.50 & 2.00 \\
\hline $\mathbf{Z H}$ & 3.00 & 3.00 & 1.50 & 3.00 & 3.00 & 13.50 & 2.00 \\
\hline AI & 3.00 & 3.00 & 3.00 & 3.00 & 3.00 & 15.00 & 2.00 \\
\hline GL & 3.00 & 3.00 & 3.00 & 3.00 & 3.00 & 15.00 & 2.00 \\
\hline
\end{tabular}

Quelle: Spörndli (1997: 32).

Zur Überprüfung des Sachwalter-Modells (Hypothese 1) verwenden wir als unabhängige Variablen die kantonalen Arbeitslosenquoten der Jahre 1990 und 1995. Wichtig ist in diesem Zusammenhang, dass sich die Aktivitäten

\footnotetext{
${ }^{14}$ Die effektiven Erwerbsquoten sind ein besserer Indikator für die Erteilung von Arbeitsbewilligungen, da in jedem Kanton ein Angebotsüberhang an arbeitswilligen Asylsuchenden besteht. Kontrollierte Erwerbsquoten müssten wir in unserer Untersuchung dann berücksichtigen, wenn auf einzelnen kantonalen Arbeitsmärkten ein Nachfrageüberhang nach Asylbewerbern existieren würde.
} 
von Asylbewerbern und vorläufig Aufgenommenen auf dem Arbeitsmarkt auf gewisse Branchen konzentrieren. Piguet und Misteli (1996: 20) stellen fest, dass 68.5 Prozent aller arbeitenden Asylbewerber entweder im Gastgewerbe (einschliesslich Restaurants) oder im Baugewerbe tätig sind. Es ist deshalb denkbar, dass die kantonale Arbeitslosigkeit in diesen Branchen in bezug auf die Restriktivität wichtiger ist als die globale Arbeitslosenquote. Wir werden diesem Umstand bei der Überprüfung der ersten Hypothese Rechnung tragen. Für das Interessengruppen-Modell operationalisieren wir die Wichtigkeit von unterdurchschnittlich produktiven und arbeitsintensiven Wirtschaftszweigen als Quote der Beschäftigten in den Wirtschaftszweigen Gastgewerbe und Baugewerbe in Relation zu allen Beschäftigten.

Tabelle 2: Die unabhängigen Variablen, ihre Indikatoren und deren Quellen

\begin{tabular}{|c|c|c|c|}
\hline Variable & Abkürzung & Indikator & Quelle \\
\hline $\begin{array}{l}\text { Arbeitslosenquote } \\
\text { (1995 und 1990) }\end{array}$ & $\begin{array}{l}\text { ALQUO } \\
\text { AL1990 }\end{array}$ & Arbeitslosenquote & $\begin{array}{l}\text { Statistisches Jahrbuch } \\
\text { d. Schweiz (1992/1997) }\end{array}$ \\
\hline $\begin{array}{l}\text { "Arbeitslosenquote" in } \\
\text { den relevanten Wirt- } \\
\text { schaftszweigen (1995) }\end{array}$ & ALWIZW & $\begin{array}{l}\text { Quote aus Arbeitslosen und Be- } \\
\text { schäftigten in den Wirtschafts- } \\
\text { zweigen "Gast-" und "Bauge- } \\
\text { wer-be" }\end{array}$ & $\begin{array}{l}\text { Arbeitslosenzahlen: } \\
\text { BIGA (1995); } \\
\text { Beschäftigtenzahlen: } \\
\text { Abrbeitsstätten (1995) }\end{array}$ \\
\hline $\begin{array}{l}\text { Wichtigkeit der rele- } \\
\text { vanten Wirtschafts- } \\
\text { zweige (1995) }\end{array}$ & GAQUO & $\begin{array}{l}\text { Beschäftigte in den Wirtschafts- } \\
\text { zweigen "Gast-" und "Bauge- } \\
\text { wer-be" als Quote der Beschäf- } \\
\text { tigten aller Wirtschaftszweige }\end{array}$ & Arbeitsstätten (1995) \\
\hline $\begin{array}{l}\text { Ausländische Bevölke- } \\
\text { rung (gesamt, 1995) }\end{array}$ & AUSL & Ausländerquote (Erwerbstätige) & $\begin{array}{l}\text { Statistisches Jahrbuch } \\
\text { der Schweiz (1997) }\end{array}$ \\
\hline $\begin{array}{l}\text { Haltung der Bevölke- } \\
\text { rung gegenüber Flücht- } \\
\text { lingen }\end{array}$ & SVPINI & $\begin{array}{l}\text { Nein-Stimmenanteil zur SVP- } \\
\text { Initiative "gegen die illegale } \\
\text { Ein-wanderung" vom 1. Dez. } \\
1996\end{array}$ & Hardmeier (1997) \\
\hline
\end{tabular}

Die Haltung der Bevölkerung in einem Kanton gegenüber Asylsuchenden messen wir mit der Zustimmung zur SVP-Initiative "gegen die illegale Einwanderung", über die am 1. Dezember 1996 abgestimmt wurde. Kantone mit einem überdurchschnittlichen Ja-Stimmenanteil lassen darauf schliessen, dass die Bevölkerung eine negative Grundeinstellung gegenüber Asylsuchenden aufweist. Die Hinzunahme von weiteren Volksabstimmungen als Indikatoren halten wir nicht für erforderlich, da die Resultate zur Asylinitiative stark mit den meisten themenverwandten Vorlagen aus den neunziger Jahren korrelieren und sich die Zustimmungsprofile in der Ausländerpolitik in räumlicher Hinsicht nicht grundsätzlich verschoben haben (Bundesamt für Statistik 1997b: 8-9). Kantonale Wahlresultate eignen sich insofern 
nicht für die Messung der Einstellung der Bevölkerung gegenüber Asylsuchenden, als die Trennlinie zwischen positiver und negativer Einstellung quer durch manche Parteien hindurch verläuft. In Tabelle 2 sind die unabhängigen Variablen unter Angabe der Datenquelle aufgelistet.

\section{Die Zustimmung zur SVP-Initiative und die Restriktivität des Vollzugs}

Bei unserer quantitativen Analyse der Bestimmungsfaktoren für die administrative Restriktivität sowie die tatsächliche Handhabung der Erteilung von Arbeitsbewilligungen an Asylsuchende verfolgen wir eine dreifache Strategie. In einem ersten Schritt berechnen wir bivariate Regressionen zwischen den beiden abhängigen Variablen und den Erklärungsfaktoren. Da sich die Kantone der Romandie insbesondere beim Restriktivitätsgrad von den Deutschschweizer Kantonen unterscheiden, führen wir die Regressionsanalysen einmal für alle 26 Kantone, dann für die 19 deutschsprachigen Stände durch. ${ }^{15}$ Aufgrund der beschränkten Fallzahl sowie bedeutender Multikollinearität zwischen den unabhängigen Variablen mussten wir auf eine multivariate Analyse verzichten. Da die Variable Restriktivitätsgrad für eine Regressionsanalyse nicht das adäquate Datenniveau aufweist, haben wir zweitens für die einzige unabhängige Variable, die sowohl für die Gesamtheit aller Kantone als auch für die Deutschschweiz signifikant ist, ein Ordered Probit Modell gerechnet. ${ }^{16}$ Als abhängige Variable verwenden wir dort die Dreiteilung des Restriktivitätsgrades in ein liberales, ein durchschnittliches und ein restriktives Lager (Tabelle 1). Drittens untersuchen wir, ob systematische Zusammenhänge zwischen den einzelnen Indikatoren des Restriktivitätsgrades und den unabhängigen Variablen bestehen. Piguet und Misteli (1996: 32ff.) weisen nach, dass nach dem Erlöschen des Arbeitsverbots die Erwerbsquote von Asylsuchenden nicht sprunghaft ansteigt. Dies würde darauf hindeuten, dass die Indikatoren der Anwendung der BVO eher ausschlaggebend für die Erwerbsquote sein sollten. Wir haben für diesen Analyseschritt alle Indikatoren dichotomisiert und bivariate Probits berechnet. ${ }^{17}$

\footnotetext{
${ }^{15}$ Die gemischtsprachigen Kantone Wallis und Freiburg sowie das Tessin ordnen wir der Romandie zu. Diese zugegebenermassen schmale empirische Basis lässt sich leider hier nicht nach gängigem Muster durch Berechnungen für weitere Jahre erweitern, da sowohl die abhängige Variable als auch latent fremdenfeindliche Strömungen der Bevölkerung über einen relevanten Zeitraum keine Varianz aufweisen.

${ }^{16}$ Für die theoretische Grundlegung siehe Greene (1993: 703-707). Ein interessantes Anwendungsbeispiel findet sich in Dustman (1996).

${ }^{17}$ Bei der Umkodierung der Indikatoren mussten wir nebst theoretischen Überlegungen auch in Betracht ziehen, dass die dichotomen Kategorien eine ähnliche Fallzahl enthalten. Das Arbeitsverbot haben wir so umkodiert, dass es überall dort, wo keine Verlängerung besteht, den Wert 0 annimmt, andernfalls wurde es mit 1 kodiert. Die Variable BVO erhält neu den Wert 0, falls sie gar nicht oder teilweise angewendet wird, und den Wert 1, falls sie integral angewendet wird. Falls Bemühungen der Arbeitgeber
} 
Die Resultate der bivariaten Regressionsanalysen sind in Tabelle $3 \mathrm{zu}-$ sammengestellt. Die Sachwalterhypothese bestätigt sich in bezug auf das administrative Verfahren bei der Erteilung von Arbeitsbewilligungen an Asylsuchende nicht. Im Gegenteil: Die Höhe der kantonalen Arbeitslosigkeit hat einen signifikant negativen Einfluss auf den Restriktivitätsgrad. Dieses Ergebnis gilt unabhängig davon, ob wir die Arbeitslosigkeit von 1990, von 1995 oder die Arbeitslosenquote in den relevanten Wirtschaftszweigen zu Grunde legen. Eine Erhöhung der Arbeitslosenquote von 1995 um ein Prozent hat ceteris paribus eine Verminderung des Restriktivitätsgrades um 1.1 Einheiten zur Folge. Dieses auf den ersten Blick erstaunliche Resultat lässt sich relativ einfach erklären. Aus Tabelle 1 ist ersichtlich, dass die Kantone der Romandie (mit Ausnahme des Wallis) durchwegs einen sehr niedrigen Restriktivitätsgrad aufweisen. ${ }^{18}$ Dies sind aber gleichzeitig diejenigen Kantone, die von der Rezession der 90er Jahre am stärksten betroffen sind und die höchsten Arbeitslosenquoten aufweisen. Das übliche Vorgehen wäre es nun, mittels einer Dummyvariable für die Zugehörigkeit eines Kantons zur Romandie zu kontrollieren. Aufgrund der hohen Multikollinearität zwischen einem solchen Dummy und der Arbeitslosenquote ist dies jedoch nicht möglich. Wir haben deshalb dieselben Regressionsanalysen unter Ausschluss der französischsprachigen Kantone berechnet.

Falls die Resultate für die Deutschschweiz stabil bleiben, können wir den Einfluss der Romandie als vernachlässigbar betrachten. Die Resultate in Spalte 3 von Tabelle 3 führen jedoch zu einer gegenteiligen Schlussfolgerung. In der Deutschschweiz besteht kein signifikanter Zusammenhang zwischen dem Restriktivitätsgrad und der Arbeitslosenquote. Dieses Ergebnis gilt auch hier unabhängig davon, welche Arbeitslosenquote zu Grunde gelegt wird. Die Sachwalterhypothese ist mithin für den Restriktivitätsgrad klar abzulehnen. Wenn wir die effektive Erteilung von Arbeitsbewilligungen an Asylsuchende, gemessen als Erwerbsquote, als abhängige Variable einbeziehen, so ist auch hier der Einfluss der Romandie unverkennbar. Die Koeffizienten der Regressionsanalysen für alle Kantone sind durchwegs signifikant und die Vorzeichen zeigen in die erwartete Richtung. Dies ist konsistent mit den Ergebnissen von Piguet und Misteli (1996). Eine Erhöhung der Arbeitslosigkeit von 1995 um ein Prozent führt ceteris paribus zu einer Reduktion der Erwerbsquote der Asylsuchenden um 2.24 Prozent. In

nicht oder nur für gewisse Branchen verlangt sind, so sind sie mit 0 kodiert, andernfalls mit 1 . Die Variable Branchenregelung ist bereits dichotom. Der Branchenwechsel erhält den Wert 0, falls ein solcher in den ersten sechs Monaten nach Erteilung einer Arbeitsbewilligung möglich ist, sonst den Wert 1.

${ }^{18} \mathrm{Ob}$ dieses Resultat aufgrund einer grösseren Aufgeschlossenheit der Kantone der Westschweiz gegenüber Flüchtlingen oder aufgrund einer Abneigung gegen eine "übermässige" Reglementierung zu Stande kommt, geht über den Gegenstand dieses Artikels hinaus. 
der Deutschschweiz hingegen scheint ein positiver Zusammenhang zu bestehen, der allerdings weit davon entfernt ist, statistisch signifikant verschieden von Null zu sein. Hier lässt sich die Sachwalterhypothese also nur aufrechterhalten, wenn wir alle Kantone in unsere Analyse einschliessen.

Tabelle 3: Bivariate Regressionsanalysen

\begin{tabular}{|c|c|c|c|c|}
\hline \multirow[b]{2}{*}{ Hypothese 1} & \multicolumn{2}{|c|}{ Restriktivitätsgrad } & \multicolumn{2}{|c|}{ Erwerbsquote } \\
\hline & $\mathrm{N}=26^{\mathrm{a}}$ & $\mathrm{N}=19^{\mathrm{b}}$ & $\mathrm{N}=26^{\mathrm{a}}$ & $\mathrm{N}=19^{\mathrm{b}}$ \\
\hline Konstante & $12.49(7.52)$ & $9.26(3.06)$ & $32.71(8.31)$ & $18.58(2.74)$ \\
\hline Arbeitslosenquote 1995 & $-1.11(-2.83)$ & $0.11 \quad(0.11)$ & $-2.24(-2.41)$ & 3.18 (1.37) \\
\hline Adj. $\mathrm{R}^{2}$ & 0.22 & -0.06 & 0.16 & 0.05 \\
\hline Konstante & $10.50(9.49)$ & $10.56(8.82)$ & $28.31(10.62)$ & $25.95(8.45)$ \\
\hline Arbeitslosenquote 1990 & $-4.16(-2.70)$ & $-2.97(-1.01)$ & $-7.65(-2.06)$ & $4.78(0.68)$ \\
\hline Adj. $R^{2}$ & 0.20 & 0.00 & 0.12 & -0.03 \\
\hline Konstante & $11.70(8.17)$ & $10.19(4.82)$ & $31.43 \quad(9.38)$ & $26.13(5.22)$ \\
\hline "Arbeitslosenquote" in & $-0.70(-2.79)$ & $-0.17(-0.31)$ & $-1.47(-2.51)$ & $0.40(0.31)$ \\
\hline \multicolumn{5}{|l|}{$\begin{array}{l}\text { Gast- gewerbe und Bau- } \\
\text { branche }\end{array}$} \\
\hline Adj. $R^{2}$ & 0.21 & -0.05 & 0.17 & -0.05 \\
\hline Hypothese 2 & $\mathrm{~N}=26^{\mathrm{a}}$ & $\mathrm{N}=19^{\mathrm{b}}$ & $\mathrm{N}=26^{\mathrm{a}}$ & $\mathrm{N}=19^{\mathrm{b}}$ \\
\hline Konstante & $8.23 \quad(4.13)$ & $11.12(5.83)$ & $28.93(6.54)$ & $32.88(7.49)$ \\
\hline $\begin{array}{l}\text { Anteil Gastgewerbe und } \\
\text { Baubranche }\end{array}$ & $0.00(0.01)$ & $-0.20(-0.90)$ & $-0.63(-1.19)$ & $-0.69(-1.35)$ \\
\hline Adj. $R^{2}$ & -0.04 & -0.01 & 0.02 & 0.04 \\
\hline Hypothese 3 & $\mathrm{~N}=26^{\mathrm{a}}$ & $\mathrm{N}=19^{\mathrm{b}}$ & $\mathrm{N}=26^{\mathrm{a}}$ & $\mathrm{N}=19^{\mathrm{b}}$ \\
\hline Konstante & $24.78(6.04)$ & $22.54(3.32)$ & $41.18 \quad(3.52)$ & $13.27(0.76)$ \\
\hline $\begin{array}{l}\text { Anteil Neinstimmen zur } \\
\text { SVP-Initiative }\end{array}$ & $-0.32(-4.08)$ & $-0.27(-1.92)$ & $-0.33(-1.47)$ & $0.29(0.36)$ \\
\hline Adj. $R^{2}$ & 0.39 & 0.13 & 0.04 & -0.02 \\
\hline Erwerbsquote & & & $\mathrm{N}=26^{\mathrm{a}}$ & $\mathrm{N}=19^{\mathrm{b}}$ \\
\hline Konstante & & & $20.59(4.88)$ & $28.79(4.93)$ \\
\hline Restriktivitätsgrad & & & $0.43(0.94)$ & $-0.13(-0.23)$ \\
\hline Adj. $\mathrm{R}^{2}$ & & & 0.04 & -0.06 \\
\hline
\end{tabular}

Das Interessengruppen-Modell (Hypothese 2) hält dem empirischen Test nicht stand. Zwischen dem Restriktivitätsgrad und der Wichtigkeit von Gastgewerbe und Baubranche in einem Kanton besteht kein Zusammenhang. In bezug auf die Erwerbsquote hat unsere Analyse ebenso kein gesichertes Ergebnis geliefert. Zudem zeigen die Vorzeichen der Koeffizienten 
nicht in die erwartete Richtung, unabhängig davon, ob wir die Romandie von der Analyse ausschliessen oder nicht. Je weniger wichtig die Branchen sind, in denen eine überdurchschnittliche Nachfrage nach Asylbewerbern besteht, desto niedriger ist tendenziell die Erwerbsquote.

Das Wiederwahlmodell hingegen scheint sich in bezug auf den Restriktivitätsgrad zu bestätigen. Je niedriger der Nein-Stimmenanteil zur SVPInitiative in einem Kanton, desto restriktiver ist das administrative Verfahren bei der Erteilung von Arbeitsbewilligungen an Asylsuchende. Ein um ein Prozent niedrigerer Nein-Stimmenanteil hat ceteris paribus einen um 0.32 Punkte höheren Restriktivitätsgrad zur Folge. Dieser Einfluss muss als beachtlich eingestuft werden. Der Nein-Stimmenanteil variiert von 40.60 Prozent (Appenzell Innerrhoden und Schwyz) bis zu 69.70 Prozent (Genf). Das Regressionsmodell impliziert eine Variation im Restriktivitätsgrad von 9.3 Punkten zwischen den beiden Extremen allein aufgrund der unterschiedlichen Zustimmung zur SVP-Initiative. Zwar ist auch hier der Einfluss der Romandie erkennbar. Die Resultate lassen sich aber auf die Deutschschweizer Kantone übertragen. Das in Hypothese 3 formulierte Argument, dass ein vergleichsweise hoher Ausländeranteil den Effekt der Zustimmung zur SVP-Initiative auf den Restriktivitätsgrad abschwächen kann, bedingt das Schätzen einer Regression mit einem Interaktionsterm zwischen den beiden Variablen. Hier tauchen wieder die Probleme von Multikollinearität und beschränkter Fallzahl auf, die es kumulativ erschweren, signifikante tStatistiken zu erhalten. Wir verzichten deshalb auf die Präsentation einer solchen Analyse. ${ }^{19}$

Der Nein-Stimmenanteil der SVP-Initiative hat hingegen keine Erklärungskraft für die Erwerbsquote der Asylsuchenden. Dies deutet darauf hin, dass die kantonalen Behörden bei der Reglementierung der Stimme des Volkes folgen, die Handhabung aber anderen Kriterien unterwerfen. Diese Vermutung wird durch die am Schluss von Tabelle 3 ausgewiesenen Resultate erhärtet, wonach der Restriktivitätsgrad in keinem Zusammenhang zur Erwerbsquote steht. Aus theoretischer Sicht ist dieses Ergebnis jedoch nicht erstaunlich, da die für die Erteilung von Arbeitsbewilligungen zuständigen Behörden (Fremdenpolizei und Arbeitsämter) keine Wiederwahlüberlegungen anstellen. ${ }^{20}$

\footnotetext{
${ }^{19}$ Die Ergebnisse einer bivariaten Regression zwischen dem Restriktivitätsgrad und dem Ausländeranteil bestätigen eine negative Beziehung: Je höher der Ausländeranteil, um so niedriger der Restriktivitätsgrad, d.h. desto liberaler ist die Zulassungspraxis für Asylsuchende in den Arbeitsmarkt.

${ }^{20}$ Dies steht im Widerspruch zu Piguet und Misteli (1996: 94). Die beiden Autoren finden einen negativen Zusammenhang zwischen der Erwerbsquote und dem Restriktivitätsgrad, nachdem sie auf die kantonale Arbeitslosenrate kontrolliert haben. Allerdings beschränkt sich ihre Analyse auf 11 Kantone. Zudem finden sich keine Hinweise auf die statistische Signifikanz ihrer Ergebnisse.
} 
Zusammenfassend lässt sich aus den Ergebnissen der Regressionsanalysen festhalten, dass nur das Wiederwahlmodell empirisch gestützt wird. Das Datenniveau des Restriktivitätsgrades erfüllt jedoch die technischen Anforderungen des linearen Regressionsmodells nur bedingt. Wir wollen deshalb die Wiederwahlhypothese einem härteren Test unterwerfen. $\mathrm{Zu}$ diesem Zweck führen wir mit dem trichotomisierten Restriktivitätsgrad als abhängiger Variable ein Ordered Probit für die Gesamtheit aller Kantone sowie für die Deutschschweiz durch. ${ }^{21}$ Dieses Analyseverfahren ist ein geeignetes Instrument, um den Einfluss verschiedener unabhängiger Variablen auf eine ordinal skalierte abhängige Variable zu messen.

Es ist zu beachten, dass die geschätzten Koeffizienten wegen der Nichtlinearität des Modells nicht unmittelbar interpretiert werden können.22 Nimmt man jedoch die marginalen Effekte, so ist die Interpretation einfach. Für die liberale Kategorie des trichotomisierten Restrikitivitätsgrades in bezug auf den Nein-Stimmenanteil zur SVP-Initiative beträgt dieser Wert 0.026 , für die restriktive Kategorie -0.033. Dies bedeutet, dass das Modell einen Kanton mit einem 10 Prozent höheren Nein-Stimmenanteil mit einer um 2.6 Prozent grösseren Wahrscheinlichkeit der liberalen Kategorie zuordnet. Äquivalent kann man sagen, dass ein Kanton mit einem um 10 Prozent niedrigeren Nein-Stimmenanteil eine um 3.3 Prozent grössere Chance hat, der restriktiven Kategorie zugeordnet zu werden. In Tabelle 4 fassen wir die effektive Zugehörigkeit im Vergleich mit der vom Modell prognostizierten Zugehörigkeit zu einer Kategorie zusammen.

Tabelle 4: Häufigkeiten der beobachteten und prognostizierten Resultate für die Variable RESKAT (alle Kantone)

\begin{tabular}{clccc}
\hline \multicolumn{5}{c}{ Prognostizierte RESK $\boldsymbol{A T}$} \\
\hline Beobachtete RESK $\boldsymbol{A T}$ & 0 & 1 & 2 & Total \\
0 & 3 & 3 & 0 & 6 \\
1 & 1 & 9 & 2 & 12 \\
2 & 0 & 4 & 4 & 8 \\
Total & 4 & 16 & 6 & 26 \\
\hline
\end{tabular}

Anmerkung: $\quad$ RESKAT $=0,1,2$ entsprechen RESGRAD $=.0$ bis 5,6 bis $10.5,11$ bis 15

Allein mit der Zustimmung zur SPV-Initiative ordnet das Modell 16 der 26 Kantone der richtigen Kategorie zu. Dies entspricht einem Anteil von 62

\footnotetext{
${ }^{21}$ Das Modell wurde mit LIMDEP, Version 7.0, geschätzt.

${ }^{22}$ Aus Platzgründen weisen wir nur den Vergleich zwischen tatsächlich festgestellter und aufgrund des Modells prognostizierter Zugehörigkeit zu einer Kategorie aus. Die gesamten Schätzergebnisse können auf Wunsch von den Autoren bezogen werden.
} 
Prozent an richtigen Zuordnungen. Ohne weitere Informationen würde das Modell alle Kantone der mittleren Kategorie zuordnen, weil dort am meisten Fälle vertreten sind und mithin für jede Beobachtung die Wahrscheinlichkeit einer richtigen Zuordnung so am grössten ist. Die Anzahl richtiger Voraussagen steigt durch das Einbeziehen des Nein-Stimmenanteils zur SVPInitiative um 33 Prozent. Zudem liegt keine der Prognosen krass daneben. Das Modell scheint deshalb trotz seiner Einfachheit ein guter Prädiktor für die Restriktivität der arbeitsmarktlichen Regelungen für Asylsuchende zu sein.

Die Wiederwahlhypothese wird auch durch das Ordered Probit bestätigt, wenn wir alle Kantone in die Analyse einbeziehen. Unter den vier restriktiven Kantonen, die das Modell der mittleren Kategorie zuordnet, befinden sich Bern und Zürich. In diesen beiden Ständen dürfte der über den Erwartungen des Modells liegende Restriktivitätsgrad u.a. auch in den Erfahrungen mit straffälligen Asylsuchenden im Drogenbereich begründet liegen. Die beiden anderen "Ausreisser" in dieser Richtung (Schaffhausen und Zug) sind nicht so leicht zu begründen. Von den sechs liberalen Kantonen werden Freiburg, Graubünden und Luzern vom Modell der mittleren Kategorie zugeordnet. Im Fall von Freiburg scheint es naheliegend, dass der Einfluss des deutschsprachigen Kantonsteils sich auf die Zustimmung zur Asylinitiative stärker auswirkt als auf die durch die mehrheitlich frankophone Kantonsregierung erlassene Restriktivität. Bei den beiden anderen Kantonen könnte ihre Stellung als klassische Tourismusregionen eine Rolle spielen, im Sinne der in Hypothese 2 formulierten Argumente.

Für die Deutschschweiz sind die Resultate weniger klar. Der Koeffizient für die SVP-Initiative hat zwar immer noch das erwartete Vorzeichen, ist aber nicht mehr signifikant verschieden von Null. Die marginalen Effekte sind etwas weniger ausgeprägt als im Fall aller Kantone. In Tabelle 5 sind die Kombinationen von beobachteten und vorausgesagten Kategoriezugehörigkeiten dargestellt.

Tabelle 5: Häufigkeiten der beobachteten und prognostizierten Resultate für die Variable RESKAT (Deutschschweiz)

\begin{tabular}{clccc}
\hline \multicolumn{5}{c}{ Prognostizierte RESKAT } \\
\hline Beobachtete RESKAT & 0 & 1 & 2 & Total \\
0 & 0 & 2 & 0 & 2 \\
1 & 0 & 7 & 2 & 9 \\
2 & 0 & 3 & 5 & 8 \\
Total & 0 & 12 & 7 & 19 \\
\hline
\end{tabular}

Anmerkung: $\quad$ RESKAT $=0,1,2$ entsprechen RESGRAD $=.0$ bis 5,6 bis $10.5,11$ bis 15 
Aus der letzten Spalte von Tabelle 5 ist ersichtlich, dass durch die Elimination der französischsprachigen Kantone in der untersten Restriktivitätskategorie nur noch zwei Kantone vertreten sind. Dies dürfte hauptsächlich dafür verantwortlich sein, dass der Einfluss der Abstimmung über die SVPInitiative nicht mehr signifikant ist. Das Modell schätzt auch eher konservativ und misst keinem Kanton diese Kategorie bei. Es ordnet aber immerhin 12 der 19 Deutschschweizer Kantone richtig ein, was einer Prognosegenauigkeit von 63\% entspricht. Ohne weitere Information würde das Modell alle Kantone in die mittlere Kategorie einordnen, weil dort die Fallzahl am grössten ist. Die Prognosegenauigkeit verbessert sich durch die Einbeziehung der SVP-Initiative mithin um 44\%. Trotz dieses etwas ambivalenten Resultats akzeptieren wir die Wiederwahlhypothese. Es ist vor allem eine latent fremdenfeindliche Haltung der Bevölkerung und nicht die Situation auf dem Arbeitsmarkt, welche die kantonalen Entscheidungsträger dazu veranlasst, die administrativen Regelungen restriktiv zu handhaben.

Als letzten Analyseschritt untersuchen wir, inwiefern die einzelnen in den Restriktivitätsgrad einfliessenden Instrumente durch die unabhängigen Variablen determiniert sind. Dies sollte uns zeigen, welche Dimensionen den kantonalen Behörden besonders grosse diskretionäre Macht zuschanzen. Tabelle 6 zeigt die Resultate von bivariaten Probit Schätzungen. Die einzelnen Instrumente wurden dichotomisiert. 
Tabelle 6: Determinanten der einzelnen Instrumente

\begin{tabular}{lrrrrr}
\hline & $\begin{array}{c}\text { Arbeits- } \\
\text { verbot }^{\mathrm{a}}\end{array}$ & BVO & $\begin{array}{c}\text { Bemühun- } \\
\text { gen Arbeit- } \\
\text { geber }\end{array}$ & $\begin{array}{c}\text { Branchen- } \\
\text { regelung }\end{array}$ & $\begin{array}{c}\text { Branchen- } \\
\text { wechsel }\end{array}$ \\
\hline Arbeitslosenquote 1995 & -0.16 & -0.03 & -0.17 & -0.11 & -0.01 \\
& $(-2.35)$ & $(-0.22)$ & $(-1.24)$ & $(-1.88)$ & $(-0.06)$ \\
Arbeitslosenquote 1990 & 0.21 & 0.00 & 0.06 & 0.13 & 0.00 \\
& -0.85 & -0.08 & -0.22 & -0.92 & -0.07 \\
Arbeitslosenquote in & $-2.47)$ & $(-0.41)$ & $(-1.09)$ & $(-1.58)$ & $(-0.36)$ \\
Gastgewerbe und Bau- & 0.26 & 0.01 & 0.04 & 0.10 & 0.01 \\
branche & $-2.37)$ & -0.00 & -0.15 & -0.06 & -0.00 \\
Anteil Gastgewerbe & 0.23 & $0.14)$ & $(-1.58)$ & $(-1.67)$ & $(-0.13)$ \\
und Baubranche & 0.02 & -0.03 & 0.09 & 0.10 & 0.00 \\
& $(0.59)$ & $(-1.11)$ & $(0.00)$ & $(0.10)$ & 0.003 \\
Anteil Nein-Stimmen & 0.01 & 0.05 & 0.00 & 0.00 & 0.03 \\
zur SVP-Initiative & -0.26 & -0.01 & -0.03 & -0.03 & 0.00 \\
& $(-1.90)$ & $(-1.23)$ & $(-2.00)$ & $(-1.90)$ & $(0.12)$ \\
& 0.13 & 0.06 & 0.15 & 0.13 & 0.00 \\
\hline
\end{tabular}

${ }^{a}$ Die einzelnen Felder der Tabelle enthalten die ersten partiellen Ableitungen der geschätzten ProbitKoeffizenten nach den unabhängigen Variablen. Sie wurden am Mittelwert der unabhängigen Variablen ausgewertet und sind an dieser Stelle analog zu normalen Regressionskoeffizienten zu interpretieren. Aus Platzgründen verzichten wir darauf, die Werte für die Konstanten auszuweisen. In Klammer stehen die Werte der t-Statistik für die ersten partiellen Ableitungen. Darunter befindet sich das Pseudo-R ${ }^{2}$. Für seine Berechnung, s. Aldrich und Nelson (1984: 57).

Zunächst einmal fällt auf, dass nur wenige Beziehungen auf dem $0.05 \mathrm{Ni}-$ veau statistisch signifikant sind. Der negative Einfluss der Arbeitslosigkeit auf die Verlängerung des Arbeitsverbots ist wiederum durch die liberale Haltung der Kantone der Romandie bedingt. Auf alle Instrumente gesehen hat erneut der Nein-Stimmenanteil zur SVP-Initiative die höchste Erklärungskraft. In 4 von 5 Fällen weist das Vorzeichen in die erwartete Richtung, und in drei Fällen ist der geschätzte Koeffizient wenigstens auf dem 0.10 Niveau statistisch signifikant verschieden von Null. Diese zusätzliche Analyse erhärtet mithin unsere bisherigen Resultate. Die Varianz in der Restriktivität der Erteilung von Arbeitsbewilligungen an Asylsuchende in den Kantonen lässt sich vor allem auf die unterschiedliche Haltung der Bevölkerung gegenüber Flüchtlingen zurückführen.

\section{Schluss}

Wir haben in diesem Artikel die kantonale Implementation der arbeitsmarktlichen Bestimmungen für Asylsuchende evaluiert. Wir haben empirische Evidenz dafür gefunden, dass der Vollzugsföderalismus in diesem Bereich zu gravierenden Prinzipal-Agent-Problemen führt. Der Gesetzgeber 
auf Bundesebene hat den Kantonen bei der Regelung der Erwerbstätigkeit von Asylsuchenden bewusst gewisse Freiheiten gelassen, damit der unterschiedlichen Situation auf den kantonalen Arbeitsmärkten Rechnung getragen werden kann. Unsere Analyse hat jedoch gezeigt, dass das kantonale Zulassungsverfahren nicht durch das Ausmass Arbeitslosigkeit, sondern durch den Einfluss von latent fremdenfeindlichen Strömungen in der Bevölkerung bestimmt wird. Die kantonalen Entscheidungsträger nutzen also ihre diskretionäre Macht, um die Chancen ihrer Wiederwahl zu erhöhen. Aus Sicht der normativen Föderalismustheorie ist die erhöhte Bürgernähe durchaus wünschenswert. In unserem Anwendungsfall ist das Ergebnis aber insbesondere darum bedenklich, weil die Asylsuchenden zufällig einem Kanton zugeteilt werden..$^{23}$ Das Gebot der Gleichbehandlung wird mithin krass verletzt. Störend wirkt auch die Kluft, die sich zwischen den Bekundungen und Taten der Behörden öffnet, auch wenn dieser Teilbefund im Einklang mit der normativen Föderalismustheorie steht. So haben die arbeitsmarktlichen Bestimmungen nichts mit der administrativen Praxis zu tun. Speziell die Entscheidungsträger in der Romandie scheinen einen Spagat vollziehen zu wollen. Ihrer liberalen administrativen Regelung, welche die Haltung der Mehrheit der Bevölkerung widerspiegelt, steht eine vergleichsweise niedrige Erwerbsquote entgegen. Dieser Zwiespalt zeigt, dass der Föderalismus nicht unbedingt mit einer erhöhten Transparenz der politischen Entscheidungen verbunden sein muss. Vielmehr ist es den Behörden möglich, sich über den Bevölkerungswillen hinwegzusetzen. Ob hier zentralstaatliche Systeme dank geringerer Transaktionskosten allenfalls mehr Klarheit schaffen können, bleibt in unserer Analyse unerörtert. Unsere Untersuchung ist aber ein zweifacher Hinweis darauf, dass das föderale Prinzip aus der Perspektive von Prinzipal-Agent-Modellen bedenkliche Strukturschwächen aufweist. Inwiefern die Erwerbsquoten für Asylsuchende mit der Lage auf dem Arbeitsmarkt variieren, konnten wir nicht thematisieren, weil wir nicht über Längsschnittdaten verfügen. Eine Forschung in diese Richtung würde mit Bestimmtheit neue Perspektiven öffnen.

\section{Bibliographie}

ABIZADEH, Sohrab and Philippe CYRENNE (1997). "On Distinguishing between Leviathan and Public Interest Governments in a Federal State", Public Choice 92: 281-299.

ABROMEIT, Heidrun (1993). "Föderalismus: Modelle für Europa", Österreichische Zeitschrift für Politikwissenschaft 22: 207-220.

\footnotetext{
${ }^{23}$ Der nach der Bevölkerungsgrösse der Kantone berechnete Verteilungschlüssel regelt einzig die Lastenverteilung zwischen den Ständen.
} 
ARBEITSSTÄTTEN und BESCHÄFTIGUNG (1995). Auswertungen BZ-95. Bern: Bundesamt für Statistik, Unternehmen und Beschäftigung. (Vorabdruck).

ALDRICH, John H. und Forrest D. NELSON (1984). Linear Probability, Logit, and Probit Models. Beverly Hills (CA): Sage.

BIGA (1995). Registrierte Arbeitslose nach Wirtschaftszweigen und Kantonen. Bern: Bundesamt für Industrie, Gewerbe und Arbeit, Abteilung Arbeitsmarkt. (Vorabdruck).

BUENO de MESQUITA, Bruce and David LALMAN (1992). War and Reason: Domestic and International Imperatives. New Haven (CT): Yale University Press.

BUNDESAMT für STATISTIK (1992). Statistisches Jahrbuch der Schweiz. Bern: Birkhäuser.

BUNDESAMT für STATISTIK (1997a). Statistisches Jahrbuch der Schweiz. Bern: Birkhäuser.

BUNDESAMT für STATISTIK (1997b). "Die Volksinitiative 'gegen die illegale Einwanderung"”, BFS aktuell 17. Feb.: 2-9.

BUSSMANN, Werner (1986). Mythos und Wirklichkeit der Zusammenarbeit im Bundesstaat: Patentrezept oder Sackgasse? Bern: Haupt. (Res publica Helvetica, 19).

DELLEY, Jean-Daniel (1984). "La mise en oeuvre des politiques publiques", in Ulrich KLÖTI (Hrsg.). Handbuch Politisches System der Schweiz. Bd. 2: Strukturen und Prozesse. Bern: Haupt, pp. 341-363.

DEUERLEIN, Ernst (1972). Föderalismus. Die historischen und philosophischen Grundlagen des föderativen Prinzips. Bonn: Bundeszentrale für politische Bildung.

DUSTMAN, Christian (1996). "The Social Assimilation of Immigrants", Journal of Population Economics 9: 37-54.

FAGANINI, Hans Peter (1991). Föderalistischer Aufgabenverbund in der Schweiz. Bern: Haupt.

FREY, Bruno S. und Iris BOHNET (1993). "Democracy by Competition: Referenda and Federalism in Switzerland", Publius 23: 71-81.

FREY, Bruno S. und Gebhard KIRCHGÄSSNER (1994). Demokratische Wirtschaftspolitik: Theorie und Anwendung. München: Vahlen.

FREY, René L. (1977). Zwischen Föderalismus und Zentralismus: Ein volkswirtschaftliches Konzept des schweizerischen Bundesstaates. Bern: Herbert Lang.

GERMANN, Raimund E. (1975). Vollzugsföderalismus als Forschungsobjekt. Zürich: Forschungsstelle für politische Wissenschaft, Universität Zürich.

GERMANN, Raimund E. (1986). "Die Beziehungen zwischen Bund und Kantonen im Verwaltungsbereich", in Raimund E. GERMANN und Ernest WEIBEL (Hrsg.). Handbuch Politisches System der Schweiz. Bd. 3: Föderalismus. Bern: Haupt, pp. 343-367.

GREENE, William H. (1993). Econometric Analysis. New York: Macmillan. (2nd Edition).

HARDMEIER, Sibylle (1997). Analyse der eidgenössischen Abstimmung vom 6. Dezember 1996. VOX Nr. 60. Zürich: GfS; IPZ.

HOLZER, Thomas und Gerald SCHNEIDER (1997). Convergence Towards Exclusion. Unveröffentlichtes Manuskript, Universität Konstanz.

HOLZER, Thomas, SCHNEIDER, Gerald und Thomas WIDMER (1997). Assaulting the Asylum Regime: The Impact of Legislative Deterrence Measures on Swiss Refugee Policy, 1986-1995. Unveröffentlichtes Manuskript, Universität Konstanz/Universität Zürich.

HOLZER, Thomas, SCHNEIDER, Gerald und Thomas WIDMER (1998). Discriminating Decentralization: Federalism and the Handling of Asylum Applications in Switzerland, 1988-1995. Unveröffentlichtes Manuskript Universität Konstanz/Universität Zürich. 
HÖPFLINGER, François und Kurt WYSS (1993). "Vollzugsprobleme der öffentlichen Sozialhilfe: Grenzen des Föderalismus im Bereich der wirtschaftlichen Existenzsicherung", Schweizerisches Jahrbuch für Politische Wissenschaft 33: 113-142.

HOWSE, Robert (1995). "Federalism, Democracy, and Regulatory Reform: a Sceptical View of the Case for Decentralization", in Karen KNOP et al. (eds.). Rethinking Federalism: Citizens, Markets and Governments in a Changing World. Vancouver: UBC Press, pp. 273-293.

HUEGLIN, Thomas O. (1995). "New Wine in Old Bottels? Federalism and Nation States in the Twenty-First Century: a Conceptual Overview", in Karen KNOP et al. (eds.). Rethinking Federalism: Citizens, Markets and Governments in a Changing World. Vancouver: UBC Press, pp. 203-223.

KILPER, Heiderose und Roland LHOTTA (1996). Föderalismus in der Bundesrepublik Deutschland. Opladen: Leske + Budrich.

KISSLING-NÄF, Ingrid und Peter KNOEPFEL (1992). "Politikflexibilität dank zentralstaatlichem Immobilismus? Handlungsspielräume kantonaler Vollzugspolitiken im schweizerischen politisch-administrativen System", in Heidrun ABROMEIT und Werner W. POMMEREHNE (Hrsg.). Staatstätigkeit in der Schweiz. Bern: Haupt, pp. 43-69.

KORITANSKY, John C. (1987). "Decentralization and Civic Virtue in Tocqueville's "New Science of Politics"', in Daniel J. ELAZAR (ed.). Federalism as Grand Design: Political Philosophers and the Federal Principle. Boston (MA): University Press of America, pp. 179198.

KREPS, David M. (1990). A Course in Microeconomic Theory. New York (NY): Harvester Wheatsheaf.

LEONARDY, Uwe (1996). "The Political Dimension, German Practice, and European Perspective", in Joachim Jens HESSE und Vincent WRIGHT (eds.). Federalizing Europe? The Costs, Benefits, and Preconditions of Federal Political Systems. New York: Oxford University Press, pp. 73-100.

LINDER, Wolf (1987). Politische Entscheidung und Gesetzesvollzug in der Schweiz. Bern: Haupt.

LINDER, Wolf (1994). Swiss Democracy: Possible Solutions to Conflict in Multicultural Societies. London: Macmillan.

LINDER, Wolf (1996). "Schweizerischer und europäischer Föderalismus - Gemeinsamkeiten und Unterschiede", in Wolf LINDER, Prisca LANFRANCHI und Edwald R. WEIBEL (Hrsg.). Schweizer Eigenart - eigenartige Schweiz. Bern: Haupt, pp. 181-197.

MONEY, Jeannette (1997). "No Vacancy: The Political Geography of Immigration Control", International Organization 51(4): 685-720.

NEIDHART, Leonhard (1992). "Grundlagen und Besonderheiten des schweizerischen Systems", in Heidrun ABROMEIT und Werner W. POMMEREHNE (Hrsg.). Staatstätigkeit in der Schweiz. Bern: Haupt, pp. 15-42.

NÜSSLI, Kurt (1985). Föderalismus in der Schweiz: Konzepte, Indikatoren, Daten. Grüsch: Rüegger.

PIGUET, Etienne und Roland MISTELI (1996). L'intégration des requérants d'asile et des réfugiés sur le marché de travail. Neuchâtel: Université de Neuchâtel, Forum suisse pour l'étude des migrations (FSM). (Rapport de recherche phases I et II).

SCHNEIDER, Gerald (1997). "Auswege aus der Verflechtungsfalle? Eine Analyse der wichtigsten Reformoptionen", in Thomas KÖNIG, Elmar RIEGER und Hermann SCHMITT (Hrsg.). Mannheimer Jahrbuch für Europäische Sozialforschun, Bd. 2. Frankfurt: Campus, pp. 163-177.

SPÖRNDLI, Markus (1997). Asyl und Arbeit. Seminararbeit, Institut für Politikwissenschaft, Universität Bern. 
TANNER, Egon (1982). Ökonomisch optimale Aufgabenteilung zwischen den staatlichen Ebenen. Bern: Peter Lang.

TIROLE, Jean (1988). The Theory of Industrial Organization. Cambridge (MA): MIT Press.

WÄLTI, Sonja (1996). "Insitutional Reform of Federalism: Changing the Players Rather than the Rules of the Game", Swiss Political Science Review 2: 113-141.

\section{Au service de trois maîtres? Les administrations cantonales et la variété des dispositions proposée sur le marché du travail au re- gard du droit d'asile suisse}

Un des multiples avantages souvent cités du fédéralisme est la flexibilité avec laquelle les prescriptions du centre sont appliquées dans les différentes régions de la périphérie. Dans le présent article, nous nous proposons d'examiner cette thèse à la lumière du droit d'asile. L'idée de départ est que la délégation d'exécution dont jouissent les cantons conduit à des problèmes du type «principal-agent» et soumet les autorités cantonales aux exigences de trois interlocuteurs l'administration fédérale, la population indigène et les groupes d'intérêts régionaux. Une analyse comparative quantitative montre que le degré plus ou moins restrictif, en matière d'ouverture du marché de l'emploi, des normes cantonales d'application de la loi fédérale sur l'asile n'est pas en premier lieu fonction de la situation économique comme l'aurait souhaité le législateur - mais est déterminé par l'influence des milieux xénophobes présents dans les cantons. La pratique de distribution des permis de travail aux candidats à l'asile n'a en revanche aucun rapport avec la sévérité affichée par la législation et est fonction, plus particulièrement en Suisse romande, de la situation du marché du travail. Même lorsque l'influence des groupes d'intérêts n'est pas démontrable, les décideurs cantonaux «chassent pour le moins deux lièvres en même temps». Dans la mesure où les requérants d'asile sont arbitrairement placés dans les différents cantons, les chances d'intégration économique et sociale des réfugiés sont loin de respecter le principe de l'égalité de traitement.

\section{In the Service of Three Masters? Cantonal Authorities and the Variety in Enforcing Labor Regulations in the Swiss Right of Asy- lum}

One of the often praised advantages of federalism is the flexibility with which goals of the federal administration can be adapted to regional peculiarities. We examine this claim with the Swiss asylum law as the 
empirical example. Our starting point is the assumption that the delegation of implementation power to the Cantons leads to severe principalagent problems and transform the state authorities into servants of three masters - the federal administration, the own constituency and regional interest groups. Our quantitative comparison shows that the restrictivity of the Cantonal implementation rules on the labor market is in contrast to the wishes of the lawmaker not primarily influenced by the local demand for labor. On the contrary, the strength of xenophobic forces seems to be a major determinant. The restrictivity of the formal rules does, however, not influence the number of work permits that Cantonal authorities issue. This behavior is largely influenced by labor market considerations, especially in the French speaking part of Switzerland. Although we could not find a direct influence of special interest groups on the behavior of cantonal administrations, we believe that these decision makers dance at least at two different weddings. Since asylum seekers are randomly distributed to the cantons, there is a possibility that refugees face a very unequal chance of economic and social integration.

Markus SPÖRNDLI, Stud. rer. soc., Wagnerstrasse 11, CH-3007 Bern; E-mail: Markus.Sporndli.1@sm-rww.unibe.ch.

Thomas HOLZER, lic. rer. pol., Assistent, Institut für Politikwissenschaft, Universität Bern, Lerchenweg 36, CH-3000 Bern 9; E-mail: Thomas.Holzer@ipw.unibe.ch.

Gerald SCHNEIDER, Prof. Dr., Ordinarius für Internationale Politik, Fakultät für Verwaltungswissenschaft, Fach D 86, Universität Konstanz, D-78457 Konstanz; E-mail: Gerald.Schneider@uni-konstanz.de.

Paper submitted 16 December 1997; accepted for publication 24 June 1998. 

